\title{
Robust Optimization on Regional WCO-for-Biodiesel Supply Chain under Supply and Demand Uncertainties
}

\author{
Yong Zhang, Yunjian Jiang, Miner Zhong, Nana Geng, and Dandan Chen \\ School of Transportation, Southeast University, Jiangsu, Nanjing 210096, China \\ Correspondence should be addressed to Yong Zhang; zhangyong@seu.edu.cn
}

Received 23 May 2016; Accepted 16 June 2016

Academic Editor: Lu Zhen

Copyright ( 2016 Yong Zhang et al. This is an open access article distributed under the Creative Commons Attribution License, which permits unrestricted use, distribution, and reproduction in any medium, provided the original work is properly cited.

\begin{abstract}
This paper aims to design a robust waste cooking oil- (WCO-) for-biodiesel supply chain under WCO supply and price as well as biodiesel demand and price uncertainties, so as to improve biorefineries' ability to cope with the poor environment. A regional supply chain is firstly introduced based on the biggest WCO-for-biodiesel company in Changzhou, Jiangsu province, and it comprises three components: WCO supplier, biorefinery, and demand zone. And then a robust mixed integer linear model with multiple objectives (economic, environmental, and social objectives) is proposed for both biorefinery location and transportation plans. After that, a heuristic algorithm based on genetic algorithm is proposed to solve this model. Finally, the 27 cities in Yangtze River delta are adopted to verify the proposed models and methods, and the sustainability and robustness of biodiesel supply are discussed.
\end{abstract}

\section{Introduction}

China has announced that it will reach the peak of carbon emission by 2030 or so at the Economic Cooperation AsiaPacific Meeting 2014. This not only has put great pressure on China government but also calls for a diversified energy structure. Transportation sector has occupied nearly $1 / 3$ of oil consumption in China and resulted in nearly $1 / 4$ of emission worldwide; thus, it is a key to apply new energy to transportation sector. Green transportation and sustainable transportation are also an important focus of previous transport studies [1-4].

Biofuel has become one of the most promising energies [5] to replace fossil fuels in transportation sector, because of its various biomass and wide range of sources as well as huge potential for energy saving and emission reduction [6-8]. The "Technology Roadmaps: Biofuels for Transport (2012)" published by International Energy Agency (IEA) points out that the proportion of fossil fuels replaced by biofuel in the transportation sector will increase from $2 \%$ to $27 \%$ by 2050 .

In practice, China has published lots of policies to support the development of biodiesel industry, and some cities (such as Beijing, Shanghai, and Guangzhou) have launched a pilot project to use biodiesel instead of fossil fuels for buses. The biomass cost occupies nearly $75 \%-80 \%$ of the supply chain cost; thus, WCO has gained wide attentions for its lower price than other biomasses' [9]. It is suggested in the document "Biodiesel Industry Development Policy (2015)"that WCO should be the main feedstock for biodiesel production.

Although a lot of biorefineries are set up by the support of Chinese government before 2012, most of them have gone out of production due to both feedstock supply and biodiesel demand limitations until now. For one thing, WCO price has gone up and biorefineries have suffered from short WCO supply, due to the competition of WCO-for-biodiesel and WCO-for-gutter oils industry. For another thing, biodiesel price is failing and biodiesel demand is still low, because of the public's low willingness to pay for biodiesel and the failing price of fossil fuels. Therefore, there is an urgent call for the study on optimizing WCO-for-biodiesel supply chain with uncertainties to improve biorefineries' ability to cope with the poor environment (such as failing biodiesel price, increasing WCO price, and lack of WCO or biodiesel demand).

\section{Literature Review}

WCO-for-biodiesel conversion has proved to be feasible for both economic and environmental considerations. Ou et 
al. [10] adopted life-cycle-analysis to calculate the energy consumption and greenhouse gas emission for the six biofuel pathways in China and found the WCO-for-biodiesel pathway can reduce both fossil fuel consumption and GHG emissions. Iglesias et al. [11] analyzed the environmental impact of WCO-for-biodiesel conversion and concluded that centralized production was more suitable for small territories, while decentralization was more advisable as the territory increased in area. Kelloway et al. [12] performed technical-economic analysis on the small-scale production of biodiesel from soybean and WCO; the results show that the use of soybean to produce biodiesel is unfeasible when the production is smallscale, while the adoption of WCO is feasible. SheinbaumPardo et al. [13] evaluated the potential use of WCO-forbiodiesel conversion in Mexico based on the 2010 data and found the biodiesel produced from WCO could represent $1.5 \%-3.3 \%$ of petrodiesel consumption for the road transport sector and could reduce $0.51-1.02 \mathrm{Mt}$ of $\mathrm{CO}_{2}$ emission.

However, the management of WCO-for-biodiesel supply chain is full of complexity; it includes a large number of supply chain members (such as WCO collectors, logistics company, biorefinery, biodiesel sales company, and biodiesel demander), involves several processes (WCO collection, WCO pretreatment, transportation, storage, biodiesel production and sales, waste management, etc.), and has to make decisions on strategic, tactical, and operational levels (facility location, technology selection, transportation plans, inventory strategy, production plans, vehicle routing and scheduling, etc.). Therefore, the supply chain coordination and optimization problem has been one of the most important factors that limit the industrialization of WCO-for-biodiesel [14].

Until now, few studies have especially analyzed the coordination and design of WCO-for-biodiesel supply chain. Ramos et al. [9] planned the collection routes for urban WCO and the case study showed that a decrease of $13 \%$ on mileage and $11 \%$ on fleet hiring cost is achieved after planning. $\mathrm{Li}$ and $\mathrm{Hu}$ [15] studied the influence of government policy on "gutter oil" and biodiesel production from WCO and found that a punishment with at least 0.51 of WCO price should be on "gutter oil" producers to ensure the competitive edge of biodiesel production. Jiang and Zhang [16] proposed a mixed integer linear programming model for both economic and environmental optimization, so as to determine the facility location, transportation plans, and technology choice.

However, WCO-for-biodiesel supply chain is a kind of biofuel supply chain, and lots of studies optimized the biofuel supply chain from other kinds of feedstock, such as forestbased biomass [17], empty fruit bunch [18], and agricultural waste. As for a regional biofuel supply chain, the biorefineries are sometimes far away from biomass suppliers; thus, the transportation mode for biomass is no longer limited to one mode, and then a multimodal transportation network can be integrated into the supply chain design. Marufuzzaman et al. [19] designed a reliable multimodal transportation network for biofuel supply chain when the intermodal hubs are subject to site dependent probabilistic disruptions, and the results show that it is more reasonable to adopt multimodal transportation when the disruption probability is low. Zhang et al. [17] designed a forest-based biofuel supply chain with road and rail transportation to minimize the supply chain cost and concluded that rail transportation plays role in the long-distance transportation, while the road transport is adopted for the short distance transportation.

As mentioned before, biofuel supply chain is full of uncertainties, such as biomass supply uncertainties, transportation and logistics uncertainties, production and operation uncertainties, demand and price uncertainties, and policies uncertainties [6]. Therefore, a new trend of the recent studies is to optimize the biofuel supply chain with uncertainties, mostly via stochastic optimization, fuzzy optimization, and robust optimization $[20,21]$. A robust supply chain has the ability to ensure sustainable development when dealing with both internal and external uncertainties $[22,23]$. In general, a robust supply chain is not simply to pursuit the supply chain benefits (such as profit, cost, and emission). It is a choice to balance the benefit and ability to deal with uncertainties. Foo et al. [18] proposed a robust linear programming model to design flexible empty fruit bunch allocation networks under multiple biomass supply scenarios. Tong et al. [20] tried to take advantage of the existing petroleum infrastructure in biofuel supply chain and proposed a robust mixed integer linear programming model with demand and supply uncertainty. Shabani and Sowlati [21] analyzed the effect of biomass water content uncertainty on biomass power plant's economic benefit and established a multiperiod stochasticrobust hybrid model to determine the biomass allocation and inventory.

All in all, there are few studies for the design of robust biofuel supply chain, and most of these studies just take economic objective into consideration. Thus, it is still a blank to take multiple objectives (such as economic, environmental, and social objectives) into the design of robust biofuel supply chain. This paper aims to fulfill this blank and proposes a robust mixed integer linear model with multiple objectives (economic, environmental, and social objectives) under supply and demand uncertainty.

\section{Problem Statement}

3.1. Explanation of the Regional WCO-for-Biodiesel Supply Chain. This paper abstracts a regional WCO-for-biodiesel supply chain based on the biggest WCO-for-biodiesel company in Changzhou, Jiangsu province, and its process is illustrated in Figure 1. WCO suppliers, biorefineries, and demand zones are regarded as the three-level supply chain, and its process can be explained as follows: (a) several WCO recycle sites (or WCO suppliers in this paper) are chosen or set up by biorefineries; (b) they purchase WCO that has been pretreated by local traders, who collect kitchen waste from restaurants or hotels and then extract WCO; (c) the purchased WCO is then transported to biorefineries to produce biodiesel via ship or road transportation; (d) the biodiesel is finally delivered to the demand zones via road transportation. It should be noted that although the supply chain mode discussed in the paper is abstracted from a real case in China, it is similar to the regional biomass-for-biofuel conversions in other countries, such as America [24, 25], Canada [26], and Finland [27]. 


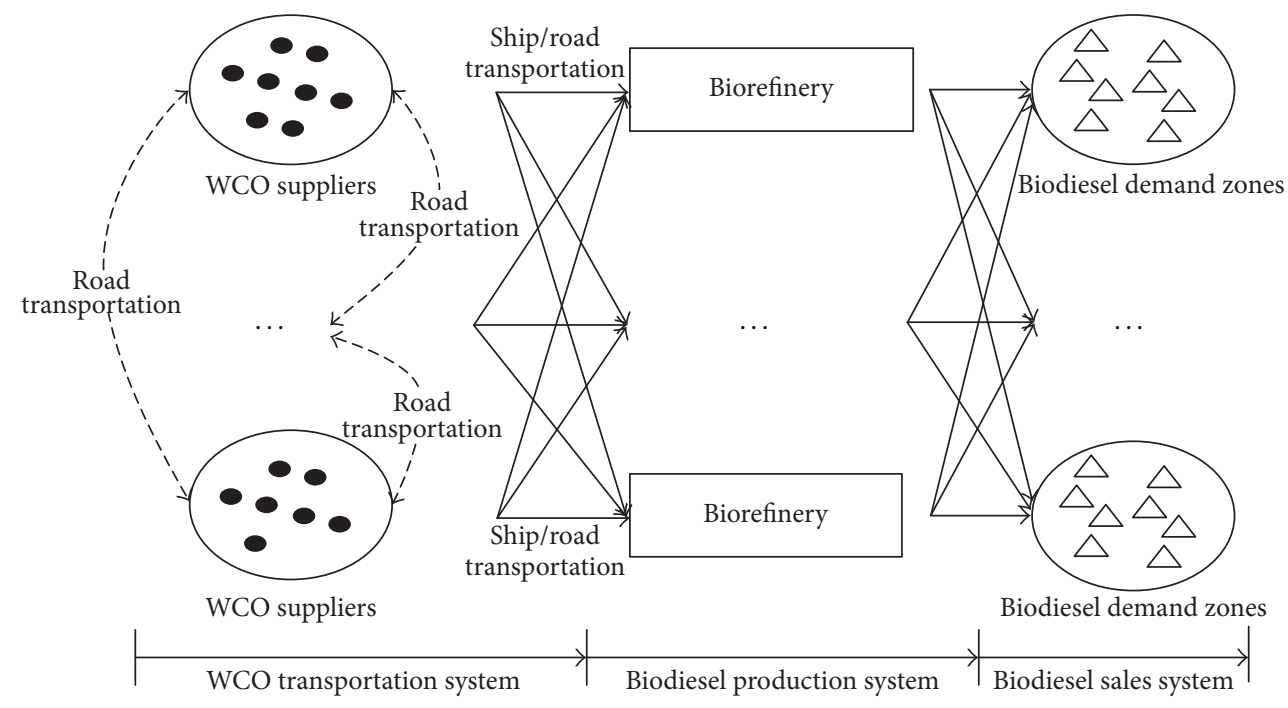

FIGURE 1: Illustration of the regional WCO-for-biodiesel supply chain.

Such a supply chain can be divided into the WCO transportation system and biodiesel production system as well as biodiesel sales system. Since the distance for WCO transportation in a region is sometimes long, the WCO is not limited to road transportation, and the ship transportation can be adopted. The selection of transportation mode can be explained as three cases: (a) when the WCO supplier is close to biorefinery, it is better to adopt road transportation; (b) when the WCO supplier is far away from the biorefinery and there exists at least one port for them, it is better to adopt ship transportation; (c) when the WCO supplier is far away from the biorefinery but there is no port for WCO supplier or biorefinery, it has to transport the WCO to other cities by road transportation first and then the ship transportation can be adopted.

As the purchased WCO has been pretreated, the process of biodiesel production is quite simple. In other words, there is only one kind of main feedstock (i.e., WCO), one kind of product (i.e., biodiesel), and one kind of technology (i.e., the WCO to biodiesel conversion). The most important in this process is the amount of biodiesel to produce, and it is denoted by the size and number of biorefineries to set up. In the biodiesel sales system, the most important problem is how to improve the profit to sell biodiesel, and it includes two problems: (a) the amount of biodiesel to sell, and it is determined by both biodiesel demand and biorefinery capacity; (b) the allocation or transportation plans for biodiesel to achieve an efficient transportation.

The regional WCO-for-biodiesel supply chain is full of uncertainties: (a) the biorefinery pays the local traders for WCO procurement; thus, WCO supply and price may be uncertain due to competitions, labor cost, and so on; (b) the capacity of biorefinery is bigger and the biodiesel demanders distribute in a large area; thus, the uncertainty of biodiesel demand is more significant; (c) the biodiesel price is highly related to diesel price which is fluctuating in recent year. Therefore, this paper tries to design a robust supply chain under WCO supply and price as well as biodiesel demand level and price uncertainties, and then three decisions are made: (a) the transportation plans of WCO; (b) the number, sizes, and locations of biorefineries; (c) the transportation plans of biodiesel. For ease of presentation, see Notations.

\subsection{Deterministic Mathematical Formulation}

3.2.1. Economic Objective Functions. Profit is the most direct and effective motivation for biorefinery, and it equals the total revenue minus the total cost:

$$
\text { profit }=\text { revenue }- \text { cost. }
$$

The revenue is the income for selling biodiesel:

$$
\text { revenue }=\mathrm{GP}^{s} \times \sum_{i \in I} \sum_{j \in J} X_{i j}^{s} .
$$

The total cost consists of five components: the discounted capital cost for biorefinery construction (denoted by FFC), as well as the procurement cost for $\mathrm{WCO}\left(\right.$ denoted by $\left.\mathrm{BFC}^{s}\right)$, the transportation cost for both WCO and biodiesel (denoted by $\mathrm{TTC}^{s}$ ), the transshipment cost for WCO (denoted by $\mathrm{LUC}^{s}$ ), and the production cost for biodiesel (denoted by $\mathrm{BPC}^{s}$ ) in each scenario $s$ :

$$
\begin{aligned}
\text { cost } & =\mathrm{FFC}+\mathrm{LUC}^{s}+\mathrm{BFC}^{s}+\mathrm{TTC}^{s}+\mathrm{BPC}^{s}, \\
\mathrm{FFC} & =\frac{1}{N} \sum_{g \in G} \sum_{i \in I} Z_{i g} \mathrm{FC}_{i g}, \\
\mathrm{BFC}^{s} & =\sum_{i \in I} \sum_{j \in J} \mathrm{MP}^{s} \times \frac{1}{\theta} \times X_{i j}^{s}, \\
\mathrm{TTC}^{s} & =\sum_{i \in I} \sum_{i \in I} \sum_{i^{\prime} \in I} c_{t i^{\prime} i} X_{t i^{\prime} i}^{s}+\sum_{i \in I} \sum_{j \in J} c_{i j} X_{i j}^{s}, \\
\mathrm{LUC}^{s} & =\sum_{i \in I_{i^{\prime} \in I}} \mathrm{UC} \times X_{1 i^{\prime} i}^{s}, \\
\mathrm{BPC}^{s} & =\sum_{i \in I} \sum_{j \in J} \mathrm{MC} \times X_{i j}^{s} .
\end{aligned}
$$



follows:

Therefore, the economic objective can be expressed as

$$
\begin{aligned}
& \max \text { profit }=\mathrm{GP}^{s} \times \sum_{i \in I} \sum_{j \in J} X_{i j}^{s}-\left[\frac{1}{N} \sum_{g \in G} \sum_{i \in I} Z_{i g} \mathrm{FC}_{i g}\right. \\
& +\sum_{i \in I} \sum_{j \in J} \mathrm{MP} \times \frac{1}{\theta} \times X_{i j}^{s}+\sum_{t \in T} \sum_{i \in I_{i^{\prime} \in I}} c_{t i^{\prime} i} X_{t i^{\prime} i}^{s} \\
& \left.+\sum_{i \in I} \sum_{j \in J} c_{i j} X_{i j}^{s}+\sum_{i \in I} \sum_{i^{\prime} \in I} \mathrm{UC} \times X_{1 i^{\prime} i}^{s}+\sum_{i \in I} \sum_{j \in J} \mathrm{MC} \times X_{i j}^{s}\right] .
\end{aligned}
$$

3.2.2. Environmental Objective Functions. The Well-to-Pump (WTP) stage is firstly adopted to estimate the emission of biorefinery. Herein, three stages are considered, and they are WCO transportation $\left(I_{1}\right)$, biodiesel production $\left(I_{2}\right)$, and biodiesel transportation $\left(I_{3}\right)$ stage. It should be noted that biorefinery purchase pretreated WCO directly from the collectors in the cities; thus, the emission for producing WCO from kitchen waste is ignored. To sum up, the environmental objective can be expressed as follows:

$$
\begin{aligned}
\text { emission }= & \sum_{t \in T} \sum_{i \in I_{i^{\prime} \in I}} e_{t i^{\prime} i} X_{t i^{\prime} i}^{s}+\sum_{i \in I} \sum_{i^{\prime} \in I} \mathrm{UE} \times X_{1 i^{\prime} i}^{s} \\
& +\sum_{i \in I} \sum_{j \in J} \mathrm{ME} \times X_{i j}^{s}+\sum_{i \in I} \sum_{j \in J} e_{i j} X_{i j}^{s} .
\end{aligned}
$$

This paper considers an emission allowances trading scheme adapted from Dal-Mas et al. [28]. Therefore, the environmental objective functions with emission allowances trading scheme can be expressed as follows:

$$
\begin{gathered}
\min \mathrm{EPR} \times(\mathrm{PE}-\mathrm{SE}), \\
\mathrm{emission}=\mathrm{Max} E+\mathrm{PE}-\mathrm{SE}, \\
\operatorname{Max} E=\mathrm{TG} \times(1-\gamma),
\end{gathered}
$$

where EPR is carbon emission trading price, $\mathrm{PE}$ is the amount of carbon emission allowance purchased, SE is the amount of carbon emission allowance sold, Max $E$ is carbon emission allowance for biorefinery, TG is the amount of carbon emission for producing fossil fuels, and $\gamma$ is the proportion of carbon emission allowed for producing biodiesel when compared to fossil fuel.

3.2.3. Social Objective Functions. WCO-for-biodiesel conversion not only is a waste-to-gold process but also helps to deal with the illegal edible oil in China. From the point of view of the whole society, it is meaningful to collect WCO as more as possible. Thus, this paper takes the amount of uncollected WCO as an objective that reflects the social responsibility of biorefinery:

$$
\text { min unuse }=\sum_{i \in I} W_{i}^{s}-\frac{1}{\theta} \times \sum_{i \in I} \sum_{j \in J} X_{i j}^{s} .
$$

3.2.4. WCO Supplier Constraints. Constraint (8) ensures that, for every region, the amount of WCO transported to other regions should not exceed the sum of the amounts of WCO self-supplied and transported. Constraint (9) ensures that only if there exists a link of transportation mode between two regions can the transportation plan be applied to them:

$$
\begin{gathered}
\sum_{t \in T i^{\prime} \in I} X_{t i i^{\prime}}^{s} \leq W_{i}^{s}+\sum_{t \in T i^{\prime} \in I} \sum_{t i^{\prime} i}^{s}, \quad \forall i \in I, s \in S, \\
X_{t i^{\prime} i}^{s} \leq M \times L_{t i^{\prime} i}, \quad \forall i^{\prime}, i \in I, s \in S .
\end{gathered}
$$

3.2.5. Biorefinery Constraints. Constraint (10) enforces the fact that a biorefinery should have no more than one capacity level at the same time. Constraint (11) ensures that the amount of biodiesel produced in biorefinery should not exceed its capacity. Constraints (12) and (13) limit the fact that all of the WCO transported to biorefinery should be converted into biodiesel. Constraint (14) enforces the fact that only if a biorefinery is built can the transportation plans be allocated within the region, and the amount of WCO transported should not exceed the amount the region can supply. Constraint (15) ensures that only when the biorefinery is open can the biodiesel sales plans be carried out:

$$
\begin{aligned}
& \sum_{g} Z_{i g} \leq 1, \quad \forall i \in I, \\
& \theta \times\left(\sum_{t \in T_{i^{\prime} \in I}} X_{t i^{\prime} i}^{s}-\sum_{t \in T_{i^{\prime} \in I}} X_{t i i^{\prime}}^{s}+Y_{i}^{s}\right) \\
& \quad \leq \sum_{g \in G} Z_{i g} \mathrm{CF}_{g}+\theta \times W_{i}^{s} \times\left(1-\sum_{g \in G} Z_{i g}\right),
\end{aligned}
$$

$\forall i \in I, s \in S$,

$$
\begin{aligned}
& \theta \times\left(-\sum_{t \in T_{i^{\prime} \in I^{\prime}}} X_{t i^{\prime} i}^{s}+\sum_{t \in T_{i^{\prime} \in I^{\prime}}} X_{t i i^{\prime}}^{s}-Y_{i}^{s}\right)+\sum_{j \in J} X_{i j}^{s} \\
& \leq M \times\left(1-\sum_{g \in G} Z_{i g}\right), \quad \forall i \in I, s \in S, \\
& \theta \times\left(-\sum_{t \in T_{i^{\prime} \in I}} X_{t i^{\prime} i}^{s}+\sum_{t \in T_{i^{\prime} \in I}} X_{t i i^{\prime}}^{s}-Y_{i}^{s}\right)+\sum_{j \in J} X_{i j}^{s} \geq 0,
\end{aligned}
$$$$
\forall i \in I, s \in S
$$$$
\begin{aligned}
& Y_{i}^{s} \leq W_{i}^{s} \times \sum_{g \in G} Z_{i g}, \quad \forall i \in I, j \in J, s \in S, \\
& X_{i j}^{s} \leq M \times \sum_{g \in G} Z_{i g}, \quad \forall i \in I, \quad j \in J, s \in S .
\end{aligned}
$$

3.2.6. Biodiesel Demand Zone and Other Constraints. Constraint (16) defines that all the biodiesel transported to the demand zones should not exceed its demand. Constraints (17) and (18) are decision variable constraints: 


$$
\begin{gathered}
\sum_{i \in I} X_{i j}^{s} \leq D_{j}^{s}, \quad \forall j \in J, s \in S, \\
Z_{i g} \in\{0,1\}, \quad \forall i \in I, g \in G, \\
X_{t i^{\prime} i}^{s}, X_{i j}^{s}, Y_{i}^{s} \geq 0, \quad \forall t \in T, i, i^{\prime} \in I, \quad j \in J, s \in S .
\end{gathered}
$$

3.3. Mathematical Formulation for Robust Optimization. In a robust supply chain optimization, the decisions can be divided into two parts $[5,15,29]$ : (1) decisions that should be made before uncertainties, such as locations and sizes of facilities and technology selection; (2) decisions that should be made after uncertainties, such as transportation plans and inventory strategies. Therefore, two kinds of decision variables can be identified in the robust optimization: control variable and design variable [30]. Control variables can be adjusted with uncertain data, while design variables are determined before uncertainty.

Scenario analysis method has been widely adopted to deal with supply chain uncertainties, and the wait-and-see approach is discussed most. Wait-and-see approach assumes the realization of different scenarios and then makes the decisions in each scenario in turn [31]. Mulvey et al. [32] introduced a robust linearization method when scenario is adopted to describe uncertainty firstly, and then lots of studies have improved this method. These studies have been reviewed and applied by Jabbarzadeh et al. [30]; thus, this paper adopts the robust linearization method adapted from Jabbarzadeh et al. [30].

To separate the design variable and control variables, a linear programming model can be explained as follows [33]:

$$
\begin{aligned}
\min & c^{T} x+d^{T} y, \\
& A x=b, \\
& B x+C y=e, \\
& x, y \geq 0,
\end{aligned}
$$

where $x$ denotes the vector of design variables and $y$ denotes the vector of control variables; $B, C$, and $e$ represent the uncertain parameters; $A x=b$ denotes the constraints whose coefficients are free of uncertainties; $B x+C y=e$ denotes the constraints whose coefficients are subject to uncertainties.

Robustness includes two aspects: solution robustness and model robustness. Herein, the solution robustness means the solution remains "close" to optimal for any scenario, while the model robustness means that the solution is "almost" feasible [30]. Therefore, the solution robustness can be measured by evaluating the closeness of a solution to optimality in each scenario, while the model robustness can be measured by evaluating the violations of constraints.

Define $\Omega=\{1,2, \ldots, \xi\}$ as a set of uncertain scenarios for describing the uncertainty of WCO supply and biodiesel demand as well as the price of both WCO and biodiesel, and assume each scenario occurs with probability of $p_{\xi}$; we have $\sum_{\xi \in \Omega} p_{\xi}=1$. Let $y_{\xi}$ denote the solution of control variables in scenario $\xi ; \psi_{\xi}$ represents the objective value in scenario $\xi, \delta_{\xi}$ represents the infeasibility of the model in scenario $\xi$, and $B_{\xi}, C_{\xi}$, and $e_{\xi}$ represent random variables in scenario. The robust optimization model can be formulated as follows [30]:

$$
\begin{aligned}
& \min \sum_{\xi \in \Omega} p_{\xi} \psi_{\xi} \\
& \quad+\lambda \sum_{\xi \in \Omega} p_{\xi}\left[\left(\psi_{\xi}-\sum_{\xi^{\prime} \in \Omega} p_{\xi^{\prime}} \psi_{\xi^{\prime}}\right)+2 \theta_{\xi}\right] \\
& \quad+\omega \sum_{\xi \in \Omega} p_{\xi} \delta_{\xi} \\
& \psi_{\xi}-\sum_{\xi \in \Omega} p_{\xi} \psi_{\xi}+\theta_{\xi} \geq 0, \quad \forall \xi \in \Omega \\
& \theta_{\xi} \geq 0, \quad \forall \xi \in \Omega, \\
& A x=b, \quad \forall \xi \in \Omega \\
& B_{\xi} x+C_{\xi} y_{\xi}+\delta_{\xi}=e_{\xi}, \quad \forall \xi \\
& x \geq 0, \quad y_{\xi} \geq 0, \quad \delta_{\xi} \geq 0, \quad \forall \xi \in \Omega
\end{aligned}
$$

where $\sum_{\xi \in \Omega} p_{\xi} \psi_{\xi}+\lambda \sum_{\xi \in \Omega} p_{\xi}\left[\left(\psi_{\xi}-\sum_{\xi^{\prime} \in \Omega} p_{\xi^{\prime}} \psi_{\xi^{\prime}}\right)+2 \theta_{\xi}\right]$ and $\omega \sum_{\xi \in \Omega} p_{\xi} \delta_{\xi}$ is the measure of solution robustness and model robustness, respectively. $\omega$ is used to express the tradeoff between the model robustness and solution robustness. $\lambda$ and $\theta_{\xi}$ are auxiliary variables to linearize the model.

Therefore, the robust optimization model for biodiesel supply chain optimization can be expressed as follows.

Objectives. Consider

$$
\begin{aligned}
& \min \quad-\sum_{s \in S} p_{s}\left(\mathrm{GP} \times \sum_{i \in I} \sum_{j \in J} X_{i j}^{s}-\mathrm{FFC}-\mathrm{LUC}^{s}-\mathrm{BFC}^{s}-\mathrm{TTC}^{s}-\mathrm{BPC}^{s}\right) \\
& -\lambda \sum_{s \in S} p_{s}\left[\mathrm{GP} \times \sum_{i \in I} \sum_{j \in J} X_{i j}^{s}-\mathrm{FFC}-\mathrm{LUC}^{s}-\mathrm{BFC}^{s}-\mathrm{TTC}^{s}-\mathrm{BPC}^{s}-\sum_{s^{\prime} \in S} p_{s^{\prime}}\left(\mathrm{GP} \times \sum_{i \in I j \in J} X_{i j}^{s}-\mathrm{FFC}-\mathrm{LUC}^{s}-\mathrm{BFC}^{s}-\mathrm{TTC}^{s}-\mathrm{BPC}^{s}\right)+2 \theta_{s}\right]+\omega_{1} \sum_{s \in S} p_{s} \delta_{s} \\
& \min \sum_{s \in S} p_{s}\left(\sum_{t \in T i \in I_{i^{\prime} \in I}} e_{t i^{\prime} i} X_{t i^{\prime} i}^{s}+\sum_{i \in I} \sum_{j \in J} e_{i j} X_{i j}^{s}+\sum_{i \in I i^{\prime} \in I} \mathrm{UE} \times X_{1 i^{\prime} i}^{s}+\sum_{i \in I} \sum_{j \in J} \mathrm{ME} \times X_{i j}^{s}\right) \\
& +\lambda \sum_{s \in S} p_{s}\left[\sum_{t \in T i \in I_{i^{\prime} \in I}} e_{t i^{\prime} i} X_{t i^{\prime} i}^{s}+\sum_{i \in I} \sum_{j \in J} e_{i j} X_{i j}^{s}+\sum_{i \in I_{i^{\prime} \in I}} \mathrm{UE} \times X_{1 i^{\prime} i}^{s}+\sum_{i \in I} \sum_{j \in J} \mathrm{ME} \times X_{i j}^{s}-\sum_{s^{\prime} \in S} p_{s^{\prime}}\left(\sum_{t \in T i \in I_{i^{\prime} \in I}} e_{t i^{\prime}} X_{t i^{\prime} i}^{s}+\sum_{i \in I \in J} \sum_{j \in J} e_{i j} X_{i j}^{s}+\sum_{i \in I_{i^{\prime}} \in I} \operatorname{UE} \times X_{1 i^{\prime} i}^{s}+\sum_{i \in I} \sum_{j \in J} \mathrm{ME} \times X_{i j}^{s}\right)+2 \tau_{s}\right] \\
& \min \sum_{s \in S} p_{s}\left[\sum_{i \in I} W_{i}^{s}-\frac{1}{\theta} \times \sum_{i \in I j \in J} X_{i j}^{s}\right]+\lambda \sum_{s \in S} p_{s}\left\{\sum_{i \in I} W_{i}^{s}-\frac{1}{\theta} \times \sum_{i \in I} \sum_{j \in J} X_{i j}^{s}-\sum_{s \in S} \sum_{i \in I} p_{s}\left[W_{i}^{s}-\frac{1}{\theta} \times \sum_{s \in S} \sum_{i \in I} \sum_{j \in J} p_{s} X_{i j}^{s}\right]+2 \xi_{s}\right\} .
\end{aligned}
$$


Constraints. Consider

$$
\begin{aligned}
& -\left(\mathrm{GP} \times \sum_{i \in I} \sum_{j \in J} X_{i j}^{s}-\mathrm{FFC}-\mathrm{LUC}^{s}-\mathrm{BFC}^{s}-\mathrm{TTC}^{s}\right. \\
& \left.-\mathrm{BPC}^{s}\right)+\sum_{s^{\prime} \in S} p_{s^{\prime}}\left(\mathrm{GP} \times \sum_{i \in I} \sum_{j \in J} X_{i j}^{s}-\mathrm{FFC}-\mathrm{LUC}^{s}\right. \\
& \left.-\mathrm{BFC}^{s}-\mathrm{TTC}^{s}-\mathrm{BPC}^{s}\right)+\theta_{s} \geq 0, \quad \forall s \in S, \\
& \sum_{t \in T i \in I} \sum_{i^{\prime} \in I} e_{t i^{\prime} i} X_{t i^{\prime} i}^{s}+\sum_{i \in I} \sum_{j \in J} e_{i j} X_{i j}^{s}+\sum_{i \in I^{\prime} \in I} \sum_{j E} \mathrm{UE} X_{1 i^{\prime} i}^{s} \\
& +\sum_{i \in I} \sum_{j \in J} \mathrm{ME} \times X_{i j}^{s}-\sum_{s^{\prime} \in S} p_{s^{\prime}}\left(\sum_{t \in T} \sum_{i \in I_{i^{\prime} \in I}} \sum_{t i^{\prime} i} X_{t i^{\prime} i}^{s}\right. \\
& +\sum_{i \in I} \sum_{j \in J} e_{i j} X_{i j}^{s}+\sum_{i \in I^{\prime} \in I} \sum_{j E} \times X_{1 i^{\prime} i}^{s} \\
& \left.+\sum_{i \in I} \sum_{j \in J} \mathrm{ME} \times X_{i j}^{s}\right)+\tau_{s} \geq 0, \quad \forall s \in S, \\
& \sum_{i \in I} W_{i}^{s}-\frac{1}{\theta} \times \sum_{i \in I} \sum_{j \in J} X_{i j}^{s}-\sum_{s \in S} p_{s}\left[\sum_{i \in I} W_{i}^{s}-\frac{1}{\theta}\right. \\
& \left.\times \sum_{i \in I} \sum_{j \in J} X_{i j}^{s}\right]+\xi_{s} \geq 0, \quad \forall s \in S \\
& \theta_{s}, \tau_{s}, \xi_{s}, \delta_{s} \geq 0, \quad \forall s \in S, \\
& \sum_{i \in I} X_{i j}^{s}+\delta_{s}=D_{j}^{s}, \quad \forall j \in J, s \in S
\end{aligned}
$$

(8) (15), (17) (18).

\section{Solution Procedure}

The model for a regional biofuel supply chain with multiple transportation modes is a challenging NP-hard problem [19]. Similarly, the complexity for solving the model in this paper increases rapidly with the number of network nodes (cities). Let $N$ represent the number of network nodes, let $S$ be the number of uncertain scenarios, and let $G$ be the number of grades that the biorefineries can be. Then, the possible location solutions will be $G \times 2^{N}$, the size of transportation distribution network will be $N^{2}$, and the times of transportation plans calculation will be as many as $G \times 2^{N} \times S$. When there are dozens of nodes in the regional network (such as the twentyseven nodes in the paper), the transportation plans should be calculated more than 10 million times. Therefore, a heuristic algorithm should be applied to deal with this problem.

As mentioned before, the decisions in the robust optimization should be divided into two parts: decisions before and after the realization of uncertainties. Similarly, this paper adopts a horizontal decomposition method to divide the robust supply chain optimization problem into biorefinery location problem and transportation plan problem. Then, the biorefinery location problem is solved by genetic algorithm, while the transportation plan problem is solved by CPLEX. Herein, the submodel for transportation plans in each scenario is deterministic, and the multiple objectives are transformed to single objective by weight coefficient method. The solution procedure for robust supply chain optimization is shown in Figure 2.

\section{Case Study}

It is reported that most of WCO are distributed in relatively developed cities, such as Beijing, Shanghai, Shenzhen, and Guangzhou. Yangtze River delta includes 27 cities and has been one of the most developed areas in China; thus, it is meaningful to take this area as an example to verify the proposed model. All of the cities are numbered as 1-27 and shown in Figure 3.

\subsection{Description of Case Study}

5.1.1. WCO Suppliers. As mentioned before, WCO is mainly produced from kitchen waste whose amount is highly correlated with the city population; thus, each city is regarded as the WCO suppliers [34] and the amount of WCO produced is estimated based on the population.

The proportion of WCO recycled is full of uncertainty in China. It is reported that $55 \%$ of WCO are transformed to illegal cooking oil, $37 \%$ of WCO are disposed, and merely $8 \%$ are recycled for industrial use (Respect Marketing Research Inc., 2014). However, China government has paid great attention to WCO recycling in the cities, and some cities (such as Shanghai, Nanjing, and Suzhou) have launched programs to recycle WCO produced in the restaurants, hotels, and so on. So it is reasonable to believe the proportion of WCO recycled will increase in the future. Therefore, to take these factors into consideration, this paper set the proportion of WCO recycled in positive, normal, and negative scenario to be $40 \%, 25 \%$, and $10 \%$, respectively.

Let $\lambda_{i}$ denote the population of city $i ; \bar{P}$ the average consumption of cooking oil per capita in China, which equals $12.3 \mathrm{~kg} /$ capita according to the China Statistical Yearbook 2014; $\alpha$ the average amount of WCO by consuming cooking oil per unit which varies from $20 \%$ to $30 \%$ [35] and this paper set it to be 25\%; and $\gamma^{s}$ the proportion of WCO recycled for biodiesel production in each scenario $s$. Therefore, the yield of WCO in each city $P_{i}$ can be calculated by the following and the results are shown in Table 1 :

$$
P_{i}=\lambda_{i} \times \bar{P} \times \alpha \times \gamma^{s}
$$

Driven by the rapid development of biodiesel industry and illegal cooking oil industry, the value of WCO has been gradually admitted by the society. Correspondingly, the recycling price of WCO has shown a climbing trend from $3000 \mathrm{RMB} /$ ton in 2009 to $5500 \mathrm{RMB} /$ ton in 2014 (Respect Marketing Research Inc., 2014), which exerts great pressure on biodiesel manufacturers. Thus, this paper set the WCO 


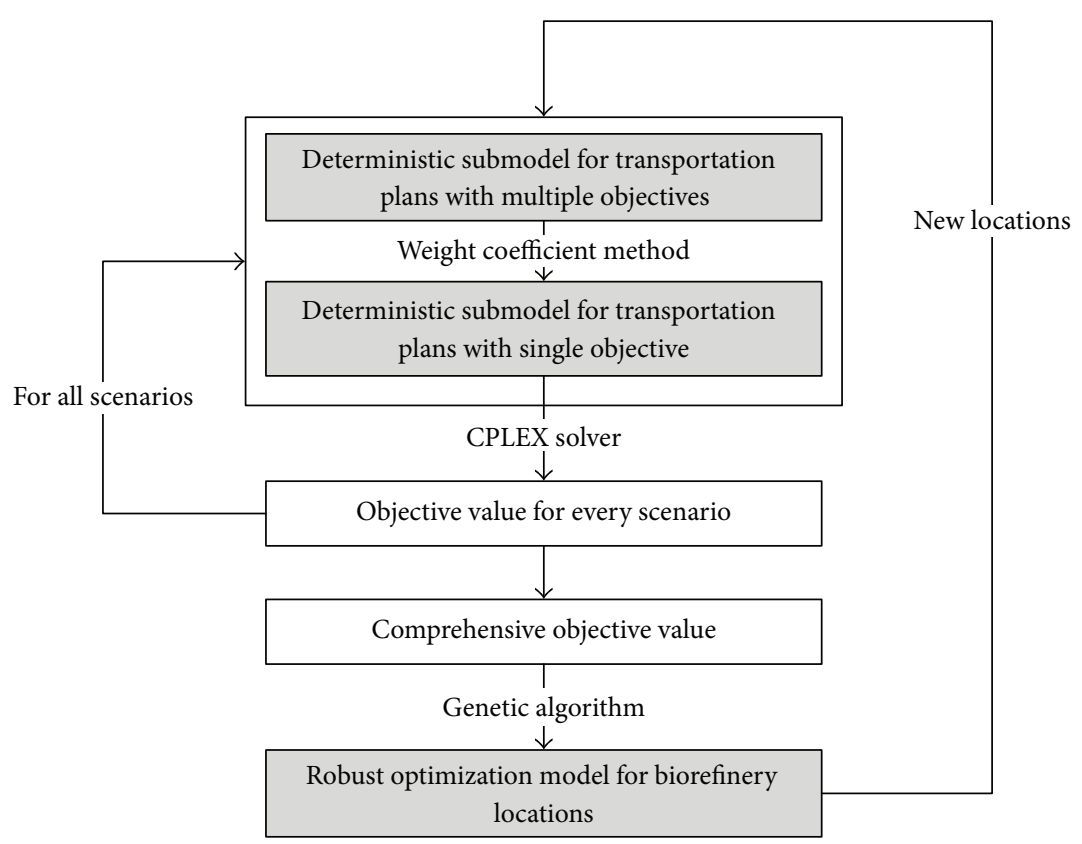

FIGURE 2: Solution procedure for robust supply chain optimization.

price in positive, normal, and negative scenario to be 3500 , 4500 , and $5500 \mathrm{RMB} /$ ton, respectively.

5.1.2. Production Facilities. China has produced more than one million tons of biodiesel annually and Jiangsu, Shandong, and Hebei province are the biggest producer. However, the annual yield of other provinces is less than 20,000 tons/year. Jiangsu Kate New Energy Company is the biggest biodiesel producer in Jiangsu whose capacity is 200,000 tons/year. Therefore, it is reasonable to set the biorefinery capacity into three grades: 50,000, 100,000, and 200,000 tons/year, and they are numbered as grades 1, 2, and 3, respectively. Similarly, the twenty-seven cities in the Yangtze River delta are chosen as the biorefinery candidates [29], and their capital costs are estimated based on regional economic development and land cost, as shown in Table 2.

5.1.3. Market Demand. Biodiesel is highly supported by the Chinese government to replace the fossil diesel for both energy-saving and environment-protecting considerations. The annual biodiesel consumption of China has increased from 560,000 tons in 2009 to 2,050,000 tons in 2014 (Respect Marketing Research Inc., 2014), and the corresponding proportion of diesel replaced by biodiesel has increased from $0.33 \%$ in 2009 to $1.19 \%$ in 2014 . Besides that, although China has launched policies to suggest that $5 \%-15 \%$ of biodiesel should be added to fossil diesel sold in refueling stations, it has no significant effect actually on the low public willingness to pay biodiesel. Thus, biodiesel demand is full of uncertainty, and the proportion of diesel replaced by biodiesel is set to be $5 \%, 2 \%$, and $1.5 \%$ for positive, normal, and negative scenario, respectively.

The twenty-seven cities chosen in the Yangtze River delta are also regarded as the demand zones for biodiesel, whose demands are estimated based on city population $[15,28]$. Let $\lambda_{i}$ denote the population of city $i ; \bar{D}$ the average consumption of diesel per capita in China, which equals $12.61 \mathrm{~kg} /$ capita according to the China Statistical Yearbook 2014; and $\beta^{s}$ the proportion of diesel replaced by biodiesel in scenario $s$. Therefore, the biodiesel demand in each city $D_{i}^{s}$ can be calculated by (24) and shown in Table 3. Besides that, the average biodiesel price ranges from 5400 to $6729 \mathrm{RMB} /$ ton during 2009 and 2014; thus, this paper set the biodiesel price in positive, normal, and negative scenario to be 5000, 6500, and $8000 \mathrm{RMB} /$ ton, respectively:

$$
D_{i}^{s}=\lambda_{i} \times \bar{D} \times \beta^{s} .
$$

5.1.4. Other Parameters. According to the biodiesel supply chain in practice, twelve typical scenarios are chosen to describe the four uncertainties in this paper, and the corresponding information is shown in Table 4. As for the other parameters in the model, they are shown in Table 5.

5.2. Results and Discussion. The model is solved by CPLEX in a personal computer Core(TM) i7-4720HQ CPU $5.19 \mathrm{GHz}$ and $4 \mathrm{G} \mathrm{RAM}$, and the results are shown as follows.

\subsubsection{Basic Results}

Comprehensive Benefits. Table 7 shows the objective values in twelve scenarios. Herein, the robust objective measures the infeasibility of the model in every scenario (actually the cost for unsatisfied biodiesel demand in this paper). As shown in Table 6, the value of comprehensive objective fluctuates greatly from -9.536 million RMB to 168.542 million RMB. Besides that, when the results of different scenarios are compared, the difference between WCO price and biodiesel 


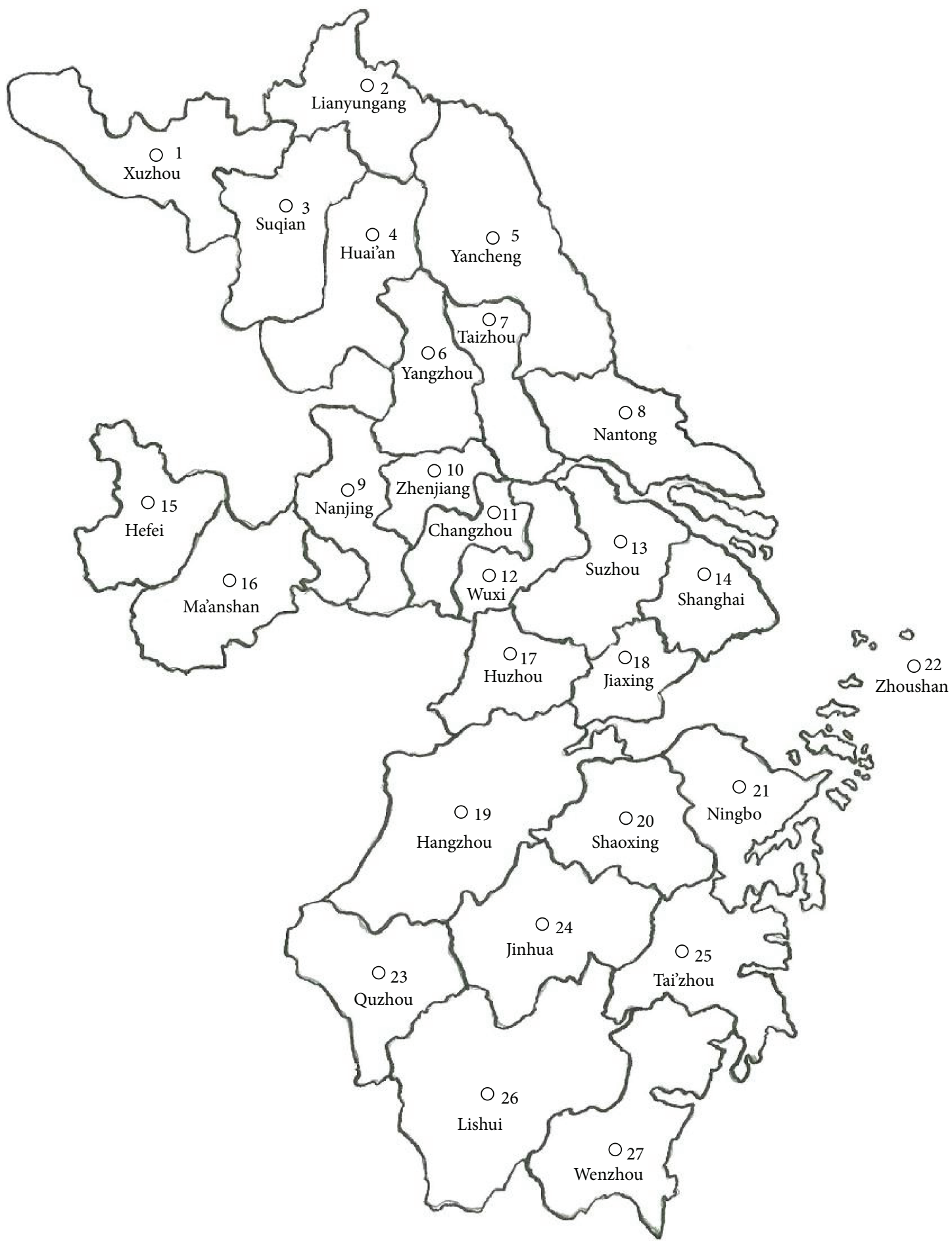

FIgURE 3: Illustration of Yangtze River delta in eastern China.

price is identified to be the dominant factor that determines the biorefinery's profit: (a) when the difference is less than $1500 \mathrm{RMB} /$ ton (scenarios 5 and 6), the biorefinery is out of production even if the WCO supply is positive; (b) when the difference is between 1500 and 2000 RMB (scenarios 7 and 11), the biodiesel demand becomes an important factor that determines whether a biorefinery is profitable or not; in other words, the biorefinery will be out of production when the biodiesel demand is low (such as scenarios 10 and 11); (c) the WCO supply also has a significant influence on biorefinery's profit when scenarios 1 and 2 are compared.

Furtherly, this paper discusses the proportion of cost and emission in each stage. As shown in Table 7, most of cost happens in WCO procurement stage, while most of carbon 
TABLE 1: Amount of collectable WCO in the regions/ton.

\begin{tabular}{lccccccccc}
\hline Collectable amount & 1 & 2 & 3 & 4 & 5 & 6 & 7 & 8 & 9 \\
\hline Positive scenario & 10613 & 5476 & 5957 & 5968 & 8884 & 5508 & 5705 & 8977 & 10106 \\
Normal scenario & 6633 & 3422 & 3723 & 3730 & 5553 & 3442 & 3566 & 5610 & 6316 \\
Negative scenario & 2653 & 1369 & 1489 & 1492 & 2221 & 1377 & 1426 & 2244 & 2526 \\
\hline Collectable amount & 10 & 11 & 12 & 13 & 14 & 15 & 16 & 17 & 18 \\
\hline Positive scenario & 3901 & 5777 & 7995 & 13043 & 29705 & 9582 & 2782 & 3244 & 4282 \\
Normal scenario & 2438 & 3610 & 4997 & 8152 & 18565 & 5989 & 1739 & 2028 & 2676 \\
Negative scenario & 975 & 1444 & 1999 & 3261 & 7426 & 2395 & 696 & 811 & 1071 \\
\hline Collectable amount & 19 & 20 & 21 & 22 & 23 & 24 & 25 & 26 & 27 \\
\hline Positive scenario & 8802 & 5449 & 7180 & 1199 & 3145 & 5843 & 7344 & 3267 & 10008 \\
Normal scenario & 5501 & 3406 & 4488 & 749 & 1965 & 3652 & 4590 & 2042 & 6255 \\
Negative scenario & 2200 & 1362 & 1795 & 300 & 786 & 1461 & 1836 & 817 & 2502 \\
\hline
\end{tabular}

TABLE 2: Capital cost of biorefineries with different grades in the regions/million RMB.

\begin{tabular}{lccccccccc}
\hline Capital cost & 1 & 2 & 3 & 4 & 5 & 6 & 7 & 8 \\
\hline Grade 1 & 12.60 & 15.12 & 15.12 & 15.12 & 12.60 & 12.60 & 22.68 & 12.60 & 12.60 \\
Grade 2 & 21.00 & 25.20 & 25.20 & 25.20 & 21.00 & 21.00 & 37.80 & 21.00 & 21.00 \\
Grade 3 & 35.00 & 42.00 & 42.00 & 42.00 & 35.00 & 35.00 & 63.00 & 35.00 & 35.00 \\
\hline Capital cost & 10 & 11 & 12 & 13 & 14 & 15 & 16 & 17 & 18 \\
\hline Grade 1 & 12.60 & 15.12 & 15.12 & 12.60 & 25.20 & 17.64 & 12.60 & 17.64 & 15.12 \\
Grade 2 & 21.00 & 25.20 & 25.20 & 21.00 & 42.00 & 29.40 & 21.00 & 29.40 & 25.20 \\
Grade 3 & 35.00 & 42.00 & 42.00 & 35.00 & 70.00 & 49.00 & 35.00 & 49.00 & 42.00 \\
\hline Capital cost & 19 & 20 & 21 & 22 & 23 & 24 & 25 & 26 \\
\hline Grade 1 & 20.16 & 12.60 & 22.68 & 12.60 & 22.68 & 12.60 & 20.16 & 15.12 & 12.60 \\
Grade 2 & 33.60 & 21.00 & 37.80 & 21.00 & 37.80 & 21.00 & 33.60 & 25.20 & 21.00 \\
Grade 3 & 56.00 & 35.00 & 63.00 & 35.00 & 63.00 & 35.00 & 56.00 & 42.00 & 35.00 \\
\hline
\end{tabular}

dioxide is emitted in biodiesel production stage. It is because the WCO purchased in the regional supply chain has been pretreated for the ease of long-distance transportation, so the biorefinery should pay more cost to those pretreated WCO, and the emission in kitchen waste-for-WCO stage is not included.

Biorefinery Locations. The results show that three biorefineries with a capacity of 50,000 tons/year should be built at Shaoxing (candidate 8), Xuzhou (candidate 17), and Suzhou (candidate 19), respectively. It should be noted that there is a biorefinery with a capacity of 200,000 tons/year in practice. Therefore, when compared with the current biorefinery locations, the biorefineries are more dispersed.

Transportation Plans. Both road and ship transportation are considered for WCO transportation, and the results are displayed in Tables 8 and 9. As shown in the tables, only one transportation mode (either road or ship transportation) is adopted in most scenarios. It is because most of the cities (except candidate 14, Lishui) in Yangtze River delta have at least one port; thus, it is more efficient to adopt the ship transportation directly. However, the road and ship transportation are adopted meanwhile in scenario 2 due to the lack of port in Lishui (candidate 14).

To furtherly analyze the influence of transportation modes on economic objective, the cases for road and ship transportation and road transportation are compared. As displayed in Figure 4, the adoption of both road and ship transportation helps to reduce the WCO transportation cost and carbon emission, to the extent of $0.64 \%-31.03 \%$ and $8.31 \%-42.68 \%$, respectively. Notably, most of these reductions are due to the replacement of road transportation by ship transportation, while the direct effect of road-water joint transportation is very small.

\subsubsection{Discussion}

Comparison of Different Optimization Objectives on Biodiesel Supply Chain Design. To explain the influence of introducing environmental and social objectives, sensitivity analysis is applied for both carbon emission trading price and uncollected WCO cost. This paper sets the carbon emission trading price and uncollected WCO cost to be 10-145 RMB/ton and 5-140 RMB/ton, respectively. Then, as shown in Figure 5, there is a linear relationship between objective value and 
TABLE 3: Annual biodiesel demand in the regions/ton.

\begin{tabular}{lccccccccc}
\hline Biodiesel demand & 1 & 2 & 3 & 4 & 5 & 6 & 7 & 8 \\
\hline Positive scenario & 5440 & 2807 & 3054 & 3059 & 4554 & 2823 & 2925 & 4601 & 5180 \\
Normal scenario & 3264 & 1684 & 1832 & 1836 & 2732 & 1694 & 1755 & 2761 & 3108 \\
Negative scenario & 1088 & 561 & 611 & 612 & 911 & 565 & 585 & 920 & 1036 \\
\hline Biodiesel demand & 10 & 11 & 12 & 13 & 14 & 15 & 16 & 17 & 18 \\
\hline Positive scenario & 2000 & 2961 & 4098 & 6686 & 15227 & 4912 & 1426 & 1663 & 2195 \\
Normal scenario & 1200 & 1777 & 2459 & 4011 & 9136 & 2947 & 856 & 998 & 1317 \\
Negative scenario & 400 & 592 & 820 & 1337 & 3045 & 982 & 285 & 333 & 439 \\
\hline Biodiesel demand & 19 & 20 & 21 & 22 & 23 & 24 & 25 & 26 \\
\hline Positive scenario & 4512 & 2793 & 3681 & 615 & 1612 & 2995 & 3765 & 1675 \\
Normal scenario & 2707 & 1676 & 2208 & 369 & 967 & 1797 & 2259 & 1005 & 3130 \\
Negative scenario & 902 & 559 & 736 & 123 & 322 & 599 & 753 & 335 & 1026 \\
\hline
\end{tabular}

TABLE 4: Twelve typical uncertain scenarios.

\begin{tabular}{|c|c|c|c|c|c|c|}
\hline Uncertainty/scenarios & Scenario 1 & Scenario 2 & Scenario 3 & Scenario 4 & Scenario 5 & Scenario 6 \\
\hline WCO supply & Normal & Negative & Positive & Normal & Positive & Normal \\
\hline WCO price & High & High & Low & Low & Low & Low \\
\hline Biodiesel demand & Positive & Positive & Negative & Normal & Negative & Negative \\
\hline Biodiesel price & High & High & Medium & Medium & Low & Low \\
\hline Probability & 0.09 & 0.05 & 0.05 & 0.09 & 0.05 & 0.05 \\
\hline Uncertainty/scenarios & Scenario 7 & Scenario 8 & Scenario 9 & Scenario 10 & Scenario 11 & Scenario 12 \\
\hline WCO supply & Positive & Normal & Normal & Normal & Negative & Positive \\
\hline WCO price & Medium & Medium & Medium & Medium & Medium & Medium \\
\hline Biodiesel demand & Normal & Positive & Normal & Negative & Negative & Positive \\
\hline Biodiesel price & Medium & Medium & Medium & Medium & Medium & Low \\
\hline Probability & 0.12 & 0.12 & 0.12 & 0.12 & 0.09 & 0.05 \\
\hline
\end{tabular}

TABLE 5: Value of other parameters in the model.

\begin{tabular}{|c|c|}
\hline Parameters & Values \\
\hline Depreciation year of biorefinery $N$ & 20 years \\
\hline Conversion rate of biodiesel from WCO $\theta$ & $90 \%[36]$ \\
\hline Production cost of biodiesel per unit MC & $1300 \mathrm{RMB} /$ ton $[37]$ \\
\hline Production emission of biodiesel per unit ME & $39.61 \mathrm{CO}_{2}$ eqv./MJ [10] \\
\hline Road transportation cost per unit & $0.35 \mathrm{RMB} /$ ton $[38]$ \\
\hline Road transportation emission per unit & $0.04795 \mathrm{~kg} \mathrm{CO}_{2}$ eqv./ton-kilometer [39] \\
\hline Ship transportation cost per unit & $0.0165 \mathrm{RMB} /$ ton $[40]$ \\
\hline Ship transportation emission per unit & $0.01733 \mathrm{~kg} \mathrm{CO}$ eqv./ton-kilometer [39] \\
\hline Transshipment cost per unit & $38 \mathrm{RMB} /$ ton $[41]$ \\
\hline Transshipment emission per unit & $0.0070 \mathrm{~kg} \mathrm{CO}{ }_{2}$ eqv./ton \\
\hline Carbon emission allowance for producing 1 ton biodiesel & $41.56 \mathrm{CO}_{2}$ eqv./ton ${ }^{\mathrm{a}}$ \\
\hline Carbon emission trading price & $20^{\mathrm{b}}$ \\
\hline Social cost for uncollected kitchen waste per unit & $5 \mathrm{RMB} /$ ton \\
\hline Robust risk coefficient $\lambda$ & 0.1 \\
\hline
\end{tabular}

${ }^{a}$ The life cycle carbon emission of fossil fuel is $103.92 \mathrm{CO}_{2}$ eqv./MJ [10]; this paper takes $40 \%$ of the carbon emission of fossil fuel as the carbon emission allowance for producing biodiesel [28].

${ }^{\mathrm{b}}$ China has carried out carbon emissions trading pilot project Beijing, Shenzhen, Shanghai, and so on, and the carbon emission trading price is about $20-$ $25 \mathrm{RMB} /$ ton. 
TABLE 6: Objective values in every scenario/thousand RMB.

\begin{tabular}{|c|c|c|c|c|c|c|}
\hline Index/scenarios & Scenario 1 & Scenario 2 & Scenario $3^{*}$ & Scenario 4 & Scenario $5^{*}$ & Scenario $6^{*}$ \\
\hline Comprehensive objective & 55,604 & 18,466 & $-9,536$ & 75,609 & $-5,440$ & $-5,066$ \\
\hline Economic objective & 55,501 & 24,139 & $-2,394$ & 75,796 & $-2,394$ & $-2,394$ \\
\hline Environmental objective & 158 & 72 & 0 & 96 & 0 & 0 \\
\hline Social objective & -55 & 0.0 & -999 & -283 & -999 & -624 \\
\hline Robust objective & 0.0 & 5,745 & 6,143 & 0.0 & 2,048 & 2,048 \\
\hline Index/scenarios & Scenario 7 & Scenario 8 & Scenario 9 & Scenario $10^{*}$ & Scenario $11^{*}$ & Scenario 12 \\
\hline Comprehensive objective & 8,211 & 100,182 & 8,032 & $-9,161$ & $-8,787$ & 168,542 \\
\hline Economic objective & 8,772 & 100,369 & 8,219 & $-2,394$ & $-2,394$ & 168,812 \\
\hline Environmental objective & 97 & 96 & 96 & 0 & 0.0 & 159 \\
\hline Social objective & -657 & -283 & -283 & -624 & -250 & -430 \\
\hline Robust objective & 0 & 0 & 0 & 6,143 & 6,143 & 0 \\
\hline
\end{tabular}

${ }^{*}$ The biorefinery is out of production in the scenario.

TABLE 7: The proportion of cost and emission in each stage.

\begin{tabular}{lcccccc}
\hline \multirow{2}{*}{ Index } & \multicolumn{2}{c}{ WCO procurement stage } & \multicolumn{2}{c}{ Biodiesel production stage } & \multicolumn{2}{c}{ Biodiesel sales stage } \\
& Cost & Emission & Cost & Emission & Cost & Emission \\
\hline Maximum & $81.59 \%$ & $0.30 \%$ & $25.43 \%$ & $99.49 \%$ & $0.95 \%$ & $0.46 \%$ \\
Minimum & $73.63 \%$ & $0.16 \%$ & $18.13 \%$ & $99.31 \%$ & $0.30 \%$ & $0.21 \%$ \\
\hline
\end{tabular}

TABLE 8: Road transportation plans of WCO in different scenarios.

\begin{tabular}{lccc}
\hline Scenario & Facility 8 & Facility 17 & Facility 19 \\
\hline 1 & $4 / 5501,8 / 3406$ & $17 / 6633,27 / 3273$ & $1 / 18565,6 / 2676,7 / 2028,16 / 4997,18 / 3610,19 / 8152,20 / 5610$ \\
$2^{*}$ & $4 / 2200,8 / 1362,11 / 1461$ & $17 / 2653,27 / 1489$ & $1 / 7426,6 / 1071,7 / 811,16 / 1999,18 / 1999,19 / 3261,20 / 2244$ \\
4 & $4 / 5501,8 / 3406$ & $17 / 6633,27 / 3273$ & $1 / 14858,6 / 2676,7 / 2028,16 / 4997,18 / 3610,19 / 8152,20 / 1239$ \\
7 & $4 / 8802,8 / 5449$ & $17 / 10613,27 / 2235$ & $1 / 3291,6 / 4282,7 / 3244,16 / 7995,18 / 5557,19 / 13043$ \\
8 & $4 / 5501,8 / 3406$ & $17 / 6633,27 / 3273$ & $1 / 14858,6 / 2676,7 / 2028,16 / 4997,18 / 3610,19 / 8152,20 / 1239$ \\
9 & $4 / 5501,8 / 3406$ & $17 / 6633,27 / 3273$ & $1 / 14858,6 / 2676,7 / 2028,16 / 4997,18 / 3610,19 / 8152,20 / 1239$ \\
12 & $4 / 8802,8 / 5449$ & $17 / 10613,27 / 5957$ & $1 / 22582,6 / 4282,7 / 3244,16 / 7995,18 / 5777,19 / 13043,20 / 5676$ \\
\hline
\end{tabular}

${ }^{*} 817$ tons of WCO is transported from region 14 to region 10 in this scenario.

TABLE 9: Ship transportation plans of WCO in different scenarios.

\begin{tabular}{|c|c|c|c|}
\hline Scenario & Facility 8 & Facility 17 & Facility 19 \\
\hline 1 & $\begin{array}{c}3 / 1739,5 / 4488,9 / 749,10 / 6255 \\
12 / 1965,13 / 4590,15 / 1060\end{array}$ & $3 / 612,22 / 3422,23 / 3730,24 / 3294$ & $\begin{array}{c}16 / 5256,24 / 2259,25 / 3442,26 / 2438 \\
27 / 3566\end{array}$ \\
\hline 2 & $10 / 3093$ & $21 / 1369,22 / 1492$ & $\begin{array}{c}2 / 2395,3 / 696,5 / 1795,9 / 300,10 / 225 \\
12 / 786,13 / 1836,15 / 2526,23 / 2221 \\
24 / 1377,25 / 975,26 / 1426\end{array}$ \\
\hline 4 & $1 / 3707,5 / 4488,9 / 749$ & $22 / 2491$ & \\
\hline 7 & $10 / 3600$ & & \\
\hline 8 & $1 / 3707,5 / 4488,9 / 749$ & $22 / 2491$ & \\
\hline 9 & $1 / 3707,5 / 4488,9 / 749$ & $22 / 2491$ & \\
\hline 12 & $1 / 7123,5 / 7180,9 / 1199$ & $22 / 4843$ & \\
\hline
\end{tabular}

uncollected WCO cost when the carbon emission trading price is fixed, and vice versa.

Comparison of Optimizations in Uncertain and Deterministic Environment. In order to analyze the influence of uncertainties on the supply chain decisions, this paper optimizes the supply chain for the most positive scenario and the medium scenario, respectively. A positive scenario means a high level of WCO supply, biodiesel demand, and price as well as a low WCO price. The results show that five biorefineries with a capacity of 50,000 tons/year are chosen in the positive scenario, and the number is more than the robust optimization's (three biorefineries with a capacity of 50,000 tons/year). 


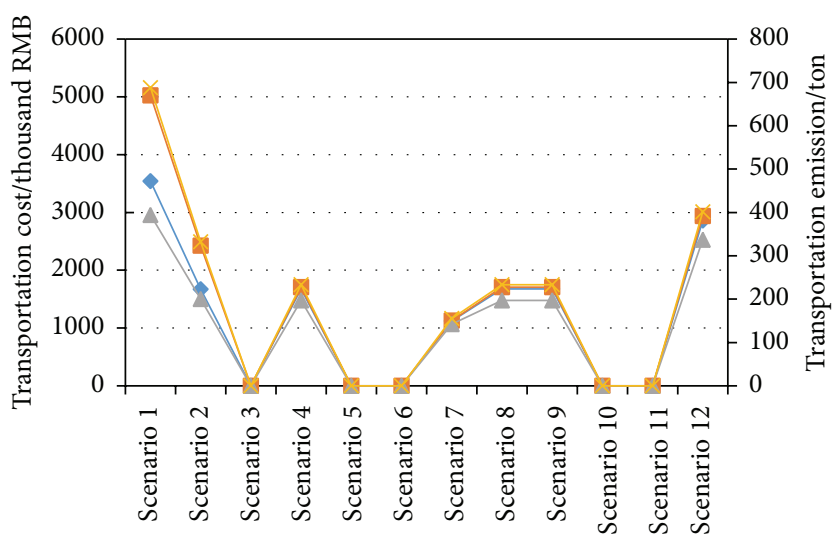

$\neg$ Transportation cost for both road and ship transportation

- - Transportation cost for road transportation only

- Transportation emission for both road and ship transportation

$\times$ Transportation emission for road transportation only

FIGURE 4: Comparison of the cost and carbon emission for different transportation modes.

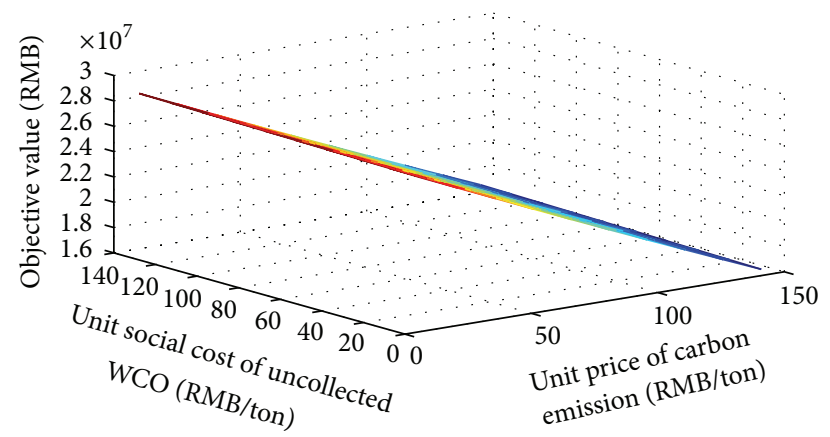

FIGURE 5: Sensitivity analysis on unit social cost of uncollected WCO and unit price of carbon emission.

As for a medium scenario, it means the level of both supply and demand side is normal. The results show that three biorefineries with a capacity of 50,000 tons/year are chosen, but the locations of these biorefineries are different from the robust optimization's. Hence, this paper concludes that uncertainties do influence the location as well as the transportation plans in biodiesel supply chain.

Furthermore, when compared with robust optimization's, the differences of the objective values in the most positive scenario and medium scenario are also discussed. As shown in Figure 6, the decisions made according to the most positive scenario have lower ability to deal with uncertainties, which results in a bigger fluctuation in different scenarios. However, the decisions made according to the medium scenario perform a close value to the robust optimizations.

\section{Conclusions}

This paper tried to improve the robustness and sustainability for regional WCO-for-biodiesel supply chain under supply and demand uncertainty. A regional supply chain including WCO suppliers, biorefineries, and demand zones

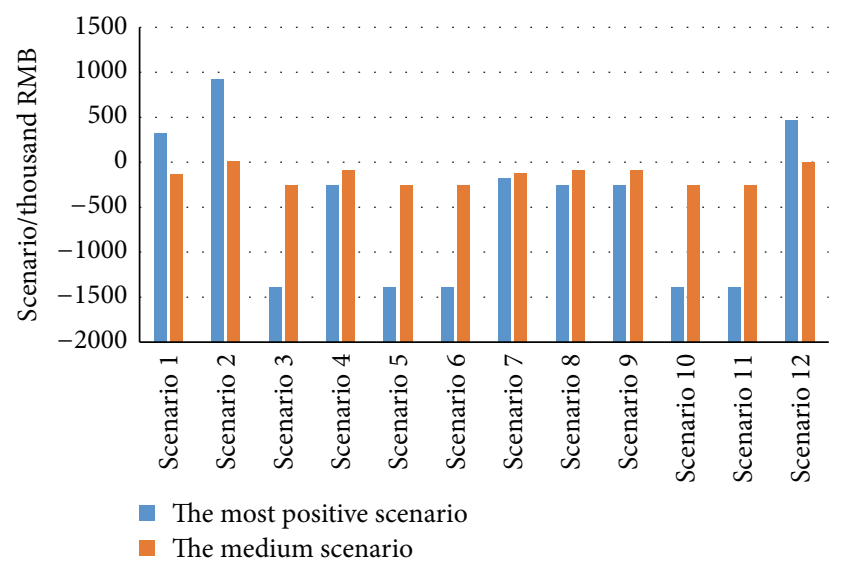

FIgURE 6: Differences of the objective values in the most positive scenario, medium scenario, when compared with robust optimizations.

in practice was firstly introduced, and then a model without uncertainties was proposed for economic (profit), environmental (carbon emission), and social (uncollected WCO) optimization. Secondly, a robust model was proposed by describing the main uncertainties (e.g., WCO supply and price as well as biodiesel demand and price uncertainty) as several uncertain scenarios. Thirdly, the genetic algorithm was applied to solve the robust model by dividing the model into several submodels through horizontal decomposition. Finally, a case study on the Yangtze River delta region was performed to verify the model efficiency, and the robustness and sustainability of biodiesel supply chain are discussed. This paper described the main research findings and gave some suggestions to support the development of biodiesel industry:

(i) All of the uncertainties (WCO supply and price as well as biodiesel demand level and price uncertainties) discussed in the model play a role in determining the biorefinery's profit which may fluctuate greatly. Therefore, the future of both feedstock and product market should be well evaluated by biorefineries and a consideration of robust decision is useful and necessary at the strategic level, especially when the market is pessimistic.

(ii) Three biorefineries with a capacity of 50,000 tons/year should be built in the optimal decisions. The decision prefers decentralized production for biodiesel when compared with the practice that has a biorefinery with a capacity of 200,000 tons/year. Therefore, a decentralized production is more profitable for biorefinery if the management convenience is considered.

(iii) The adoption of both road and ship transportation helps to reduce the WCO transportation cost and carbon emission, to the extent of $0.64 \%-31.03 \%$ and $8.31 \%-42.68 \%$, respectively. Therefore, the coordination of different transport modes (such as road transportation, ship transportation) should be well 
planned so as to ease the implementation of multiple transportation.

(iv) The introduction of environmental and social objectives does influence the biorefineries' profit; thus, some policies about the environmental and social goals can be published by the government to encourage biorefineries to go green and fulfill social responsibility.

To take both robustness and sustainability into consideration in the biofuel supply chain design stage was important and meaningful. It could not only improve biorefineries' ability to overcome supply chain uncertainties but also ensure an acceptable loss in pessimistic situation. However, this paper just tried to design a supply chain from the point of view of modelling; thus, there were some aspects to improve in the future work. On one hand, the description of sustainability was limited to three quantitative objectives, but some qualitative indicators that are not easy to measure are also important. Thus, it may be more reasonable to take both quantitative and qualitative objectives into consideration. On the other hand, this paper described the four main uncertainties by several scenarios, but the interaction between different uncertainties (such as the WCO supply and price, WCO and biodiesel price, and biodiesel demand and price) was not discussed. It may be interesting to adopt both mathematical modelling and simulation to design a robust biodiesel supply chain.

\section{Notations}

Sets

$I$ : Set of region $i$ (also set of WCO suppliers and biorefinery candidates)

$J$ : Set of biodiesel demand zone $j$

$G$ : Set of biorefinery capacity level $g$

$T$ : Set of transportation mode $t$, equal to 0 if road transportation is chosen and 1 if ship transportation is chosen

$S$ : Set of uncertain scenario $s$.

\section{Parameters}

$\mathrm{MP}^{s}$ : Price of WCO per unit in uncertain scenario $s$

$W_{i}^{s}$ : Amount of WCO that supply point $i$ can supply in uncertain scenario $s$

$D_{j}^{s}$ : Biodiesel demand of zone $j$ in uncertain scenario $s$

$\mathrm{GP}^{s}$ : Price of biodiesel per unit in uncertain scenario $s$

$N$ : Depreciation year of biorefinery

$\mathrm{CF}_{g}$ : Amount of biodiesel that a biorefinery with capacity level $g$ can produce

$\mathrm{FC}_{i g}$ : Capital cost to build a biorefinery with capacity level $g$ at candidate $i$

$\theta$ : Conversion rate of biodiesel from WCO

MC: Production cost of biodiesel per unit $c_{t i^{\prime} i}$ : Cost to transport WCO per unit from region $i^{\prime}$ to region $i$ by transportation mode $t$

$c_{t i j}$ : Cost to transport biodiesel per unit from region $i$ to demand zone $j$

UC: Cost of WCO transshipment per unit when the ship transportation is chosen

ME: Carbon emission of producing biodiesel per unit

$e_{t i^{\prime} i}$ : Carbon emission to transport WCO per unit from region $i^{\prime}$ to region $i$ by transportation mode $t$

$e_{i j}$ : Carbon emission to transport biodiesel per unit from region $i$ to demand zone $j$

UE: Carbon emission to transship WCO per unit when the ship transportation is chosen

$L_{t i i^{\prime}}$ : Equal to 1 if transportation mode $t$ exists between region $i^{\prime}$ and region $i$ and 0 otherwise

$M$ : A very big number.

\section{Decision Variables}

$Z_{i g}$ : Equal to 1 if a biorefinery with capacity level $g$ is built at candidate $i$ and 0 otherwise

$X_{t i^{\prime} i}^{s}$ : Amount of WCO transported from region $i^{\prime}$ to region $i$ by transportation mode $t$ in uncertain scenario $s$ (herein $i \neq i^{\prime}$ )

$Y_{i}^{s}$ : Amount of WCO supplied by itself $i$ in uncertain scenario $s$

$X_{i j}^{s}$ : Amount of biodiesel transported from biorefinery candidate $i$ to demand zone $j$ in uncertain scenario $s$.

\section{Competing Interests}

The authors declare that they have no competing interests.

\section{Acknowledgments}

This work was supported by National Natural Science Foundation of China (71372198) and by Science and Technology Department of Jiangsu Province, China (BY2015070-25).

\section{References}

[1] Z. Liu, Y. Yan, X. Qu, and Y. Zhang, "Bus stop-skipping scheme with random travel time," Transportation Research Part C: Emerging Technologies, vol. 35, pp. 46-56, 2013.

[2] Y. Bie, X. Gong, and Z. Liu, "Time of day intervals partition for bus schedule using GPS data," Transportation Research Part C, vol. 60, pp. 443-456, 2015.

[3] Y. Bie, Z. Liu, and L. Lu, "Optimization of coordinated signal settings for hook-turn intersections," Journal of Advanced Transportation, vol. 50, no. 2, pp. 197-213, 2016.

[4] Z. Liu, S. Wang, W. Chen, and Y. Zheng, "Willingness to board: a novel concept for modeling queuing up passengers," Transportation Research Part B, vol. 90, pp. 70-82, 2016.

[5] J. Kim, M. J. Realff, and J. H. Lee, "Optimal design and global sensitivity analysis of biomass supply chain networks for biofuels under uncertainty," Computers and Chemical Engineering, vol. 35, no. 9, pp. 1738-1751, 2011. 
[6] I. Awudu and J. Zhang, "Uncertainties and sustainability concepts in biofuel supply chain management: a review," Renewable and Sustainable Energy Reviews, vol. 16, no. 2, pp. 1359-1368, 2012.

[7] X. Qu, Q. Meng, and Z. Liu, "Estimation of number of fatalities caused by toxic gases due to fire in road tunnels," Accident Analysis and Prevention, vol. 50, pp. 616-621, 2013.

[8] X. Qu, Y. Yang, Z. Liu, S. Jin, and J. Weng, "Potential crash risks of expressway on-ramps and off-ramps: a case study in Beijing, China," Safety Science, vol. 70, pp. 58-62, 2014.

[9] T. R. P. Ramos, M. I. Gomes, and A. P. Barbosa-Póvoa, "Planning waste cooking oil collection systems," Waste Management, vol. 33, no. 8, pp. 1691-1703, 2013.

[10] X. Ou, X. Zhang, S. Chang, and Q. Guo, "Energy consumption and GHG emissions of six biofuel pathways by LCA in (the) People's Republic of China," Applied Energy, vol. 86, supplement 1, pp. S197-S208, 2009.

[11] L. Iglesias, A. Laca, M. Herrero, and M. Díaz, "A life cycle assessment comparison between centralized and decentralized biodiesel production from raw sunflower oil and waste cooking oils," Journal of Cleaner Production, vol. 37, pp. 162-171, 2012.

[12] A. Kelloway, W. A. Marvin, L. D. Schmidt, and P. Daoutidis, "Process design and supply chain optimization of supercritical biodiesel synthesis from waste cooking oils," Chemical Engineering Research and Design, vol. 91, no. 8, pp. 1456-1466, 2013.

[13] C. Sheinbaum-Pardo, A. Calderón-Irazoque, and M. RamírezSuárez, "Potential of biodiesel from waste cooking oil in Mexico," Biomass and Bioenergy, vol. 56, no. 56, pp. 230-238, 2013.

[14] Y. Zhang, X. Bao, G. Ren, X. Cai, and J. Li, "Analysing the status, obstacles and recommendations for WCOs of restaurants as biodiesel feedstocks in China from supply chain' perspectives," Resources, Conservation and Recycling, vol. 60, pp. 20-37, 2012.

[15] Q. Li and G. Hu, "Supply chain design under uncertainty for advanced biofuel production based on bio-oil gasification," Energy, vol. 74, pp. 576-584, 2014.

[16] Y. Jiang and Y. Zhang, "Supply chain optimization of biodiesel produced from waste cooking oil," Transportation Research Procedia, vol. 12, pp. 938-949, 2016.

[17] F. Zhang, D. M. Johnson, and J. Wang, "Integrating multimodal transport into forest-delivered biofuel supply chain design," Renewable Energy, vol. 93, pp. 58-67, 2016.

[18] D. C. Y. Foo, R. R. Tan, H. L. Lam, M. K. Abdul Aziz, and J. J. Klemeš, "Robust models for the synthesis of flexible palm oilbased regional bioenergy supply chain," Energy, vol. 55, pp. 6873, 2013.

[19] M. Marufuzzaman, S. D. Eksioglu, X. Li, and J. Wang, "Analyzing the impact of intermodal-related risk to the design and management of biofuel supply chain," Transportation Research Part E, vol. 69, pp. 122-145, 2014.

[20] K. Tong, F. You, and G. Rong, "Robust design and operations of hydrocarbon biofuel supply chain integrating with existing petroleum refineries considering unit cost objective," Computers \& Chemical Engineering, vol. 68, pp. 128-139, 2014.

[21] N. Shabani and T. Sowlati, "A hybrid multi-stage stochastic programming-robust optimization model for maximizing the supply chain of a forest-based biomass power plant considering uncertainties," Journal of Cleaner Production, vol. 112, pp. 32853293, 2016.

[22] S. Wang, Z. Liu, and Q. Meng, "Segment-based alteration for container liner shipping network design," Transportation Research Part B, vol. 72, pp. 128-145, 2015.
[23] S. Wang, Z. Liu, and M. G. H. Bell, "Profit-based maritime container assignment models for liner shipping networks," Transportation Research Part B: Methodological, vol. 72, pp. 5976, 2015.

[24] H. An, W. E. Wilhelm, and S. W. Searcy, "A mathematical model to design a lignocellulosic biofuel supply chain system with a case study based on a region in Central Texas," Bioresource Technology, vol. 102, no. 17, pp. 7860-7870, 2011.

[25] A. Azadeh, H. V. Arani, and H. Dashti, "A stochastic programming approach towards optimization of biofuel supply chain," Energy, vol. 76, pp. 513-525, 2014.

[26] T. Boukherroub, A. Ruiz, A. Guinet, and J. Fondrevelle, "An integrated approach for sustainable supply chain planning," Computers \& Operations Research, vol. 54, pp. 180-194, 2015.

[27] K. Natarajan, S. Leduc, P. Pelkonen, E. Tomppo, and E. Dotzauer, "Optimal locations for second generation fischer tropsch biodiesel production in Finland," Renewable Energy, vol. 62, no. 3, pp. 319-330, 2014.

[28] M. Dal-Mas, S. Giarola, A. Zamboni, and F. Bezzo, "Strategic design and investment capacity planning of the ethanol supply chain under price uncertainty," Biomass and Bioenergy, vol. 35, no. 5, pp. 2059-2071, 2011.

[29] A. Osmani and J. Zhang, "Stochastic optimization of a multifeedstock lignocellulosic-based bioethanol supply chain under multiple uncertainties," Energy, vol. 59, pp. 157-172, 2013.

[30] A. Jabbarzadeh, B. Fahimnia, and S. Seuring, "Dynamic supply chain network design for the supply of blood in disasters: a robust model with real world application," Transportation Research Part E, vol. 70, pp. 225-244, 2014.

[31] C.-W. Chen and Y. Fan, "Bioethanol supply chain system planning under supply and demand uncertainties," Transportation Research Part E, vol. 48, no. 1, pp. 150-164, 2012.

[32] J. M. Mulvey, R. J. Vanderbei, and S. A. Zenios, "Robust optimization of large-scale systems," Operations Research, vol. 43, no. 2, pp. 264-281, 1995.

[33] S. M. J. Mirzapour Al-E-Hashem, H. Malekly, and M. B. Aryanezhad, "A multi-objective robust optimization model for multi-product multi-site aggregate production planning in a supply chain under uncertainty," International Journal of Production Economics, vol. 134, no. 1, pp. 28-42, 2011.

[34] W. A. Marvin, L. D. Schmidt, S. Benjaafar, D. G. Tiffany, and P. Daoutidis, "Economic optimization of a lignocellulosic biomass-to-ethanol supply chain," Chemical Engineering Science, vol. 67, no. 1, pp. 68-79, 2012.

[35] D. Yue and F. You, "Game-theoretic modeling and optimization of multi-echelon supply chain design and operation under Stackelberg game and market equilibrium," Computers \& Chemical Engineering, vol. 71, pp. 347-361, 2014.

[36] L. Tan, "Current situation and development trend of the research and application of preparing biodiesel by Wof," Chemical Production \& Technology, vol. 14, no. 1, pp. 30-33, 2007 (Chinese).

[37] Q. Lu, Q. Zhu, and Z. He, "The research on domestic and foreign bio-diesel oil productive technology as well as its cost analysis," Petroleum \& Petrochemical Today, vol. 19, no. 5, pp. 8-13, 2011 (Chinese).

[38] Z. Liu, T. Qiu, and B. Chen, "A study of the LCA based biofuel supply chain multi-objective optimization model with multiconversion paths in China," Applied Energy, vol. 126, pp. 221234, 2014. 
[39] B. Jiang, J. Li, and X. Mao, "Container ports multimodal transport in China from the view of low carbon," The Asian Journal of Shipping and Logistics, vol. 28, no. 3, pp. 321-344, 2012.

[40] J. Geske, N. Berghout, and M. van den Broek, "Cost-effective balance between $\mathrm{CO}_{2}$ vessel and pipeline transport: part IIdesign of multimodal $\mathrm{CO}_{2}$ transport: the case of the west Mediterranean region," International Journal of Greenhouse Gas Control, vol. 33, pp. 122-134, 2015.

[41] H. G. Resat and M. Turkay, "Design and operation of intermodal transportation network in the Marmara region of Turkey," Transportation Research Part E: Logistics and Transportation Review, vol. 83, pp. 16-33, 2015. 

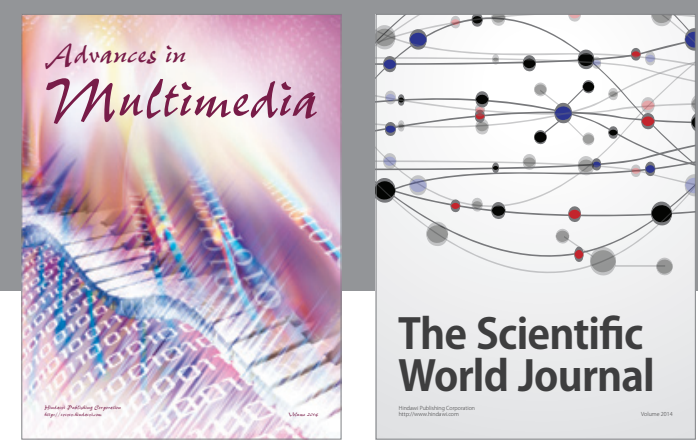

The Scientific World Journal
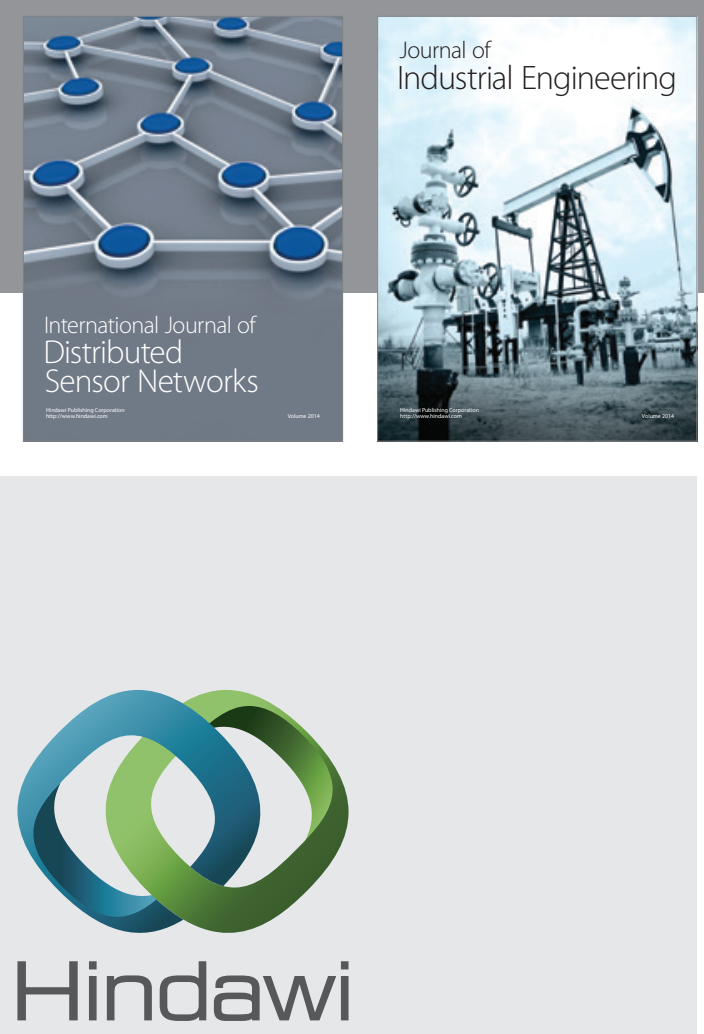

Submit your manuscripts at

http://www.hindawi.com

\section{Computer Networks} and Communications
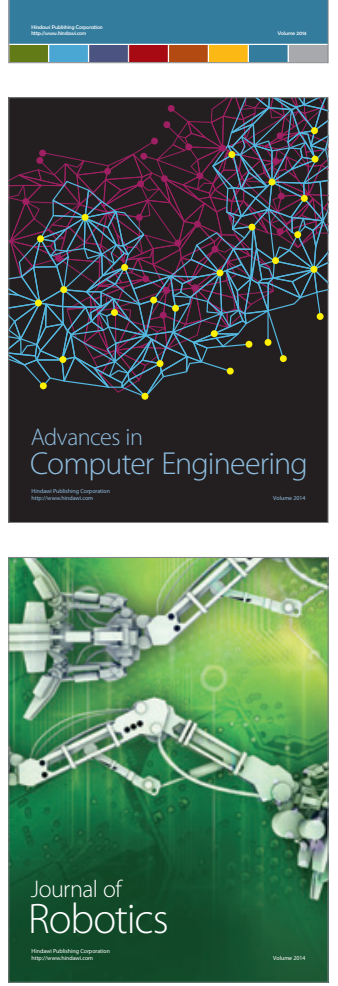
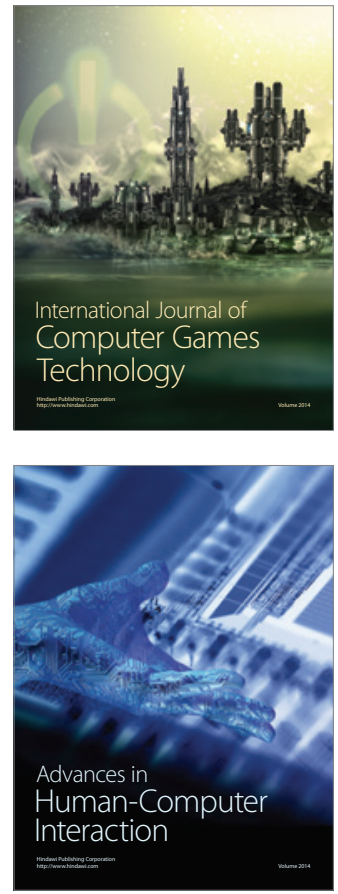
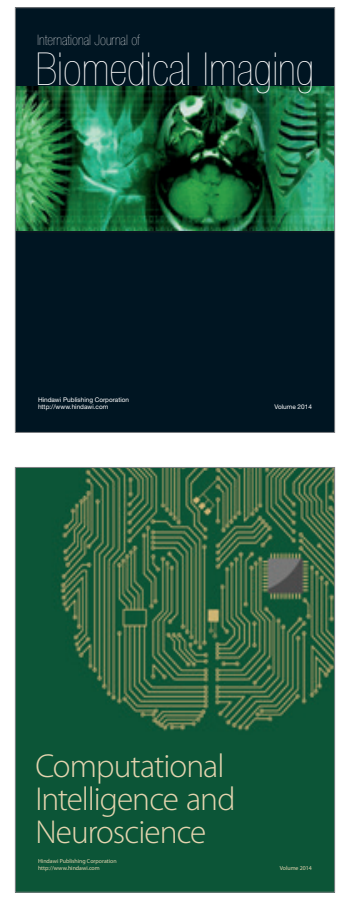
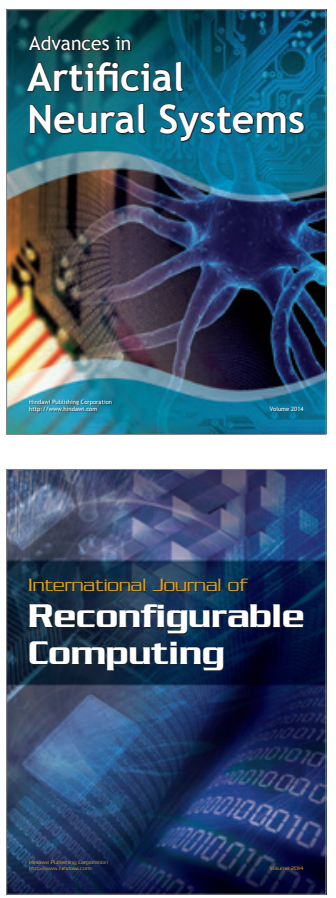
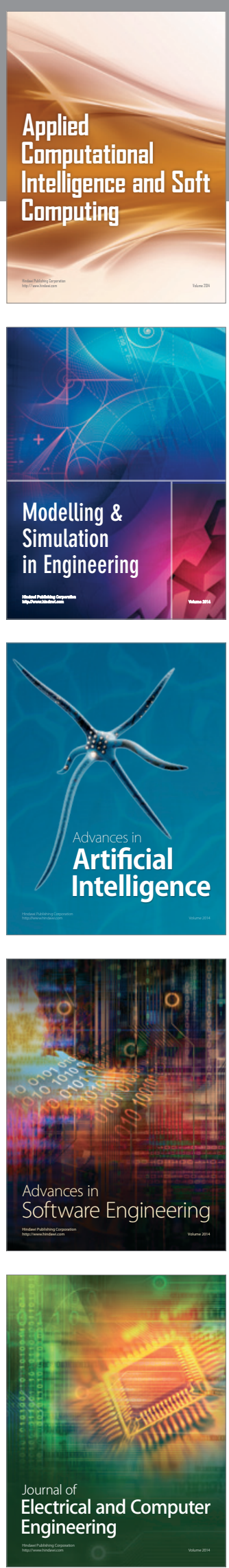